\title{
Le conflit étudiant québécois : une « épidémie » de sens pour un Québec politiquement malade
}

\section{Gabriel Blouin Genest}

\section{(2) OpenEdition}

1 Journals

\section{Édition électronique}

URL : http://journals.openedition.org/conflits/18499

DOI : 10.4000/conflits.18499

ISSN : $1777-5345$

Éditeur :

CCLS - Centre d'études sur les conflits lilberté et sécurité, L'Harmattan

\section{Édition imprimée}

Date de publication : 26 décembre 2012

Pagination : 147-151

ISBN : 978-2-336-00248-4

ISSN : 1157-996X

\section{Référence électronique}

Gabriel Blouin Genest, «Le conflit étudiant québécois : une « épidémie » de sens pour un Québec politiquement malade », Cultures \& Conflits [En ligne], 87 | Automne 2012, mis en ligne le 26 décembre 2012, consulté le 30 mars 2021. URL : http://journals.openedition.org/conflits/18499 ; DOI : https:// doi.org/10.4000/conflits.18499 


\section{Le conflit étudiant québécois : une " épidémie » de sens pour un Québec politiquement malade}

\section{Gabriel BLOUIN GENEST}

Gabriel Blouin Genest vient de compléter une thèse de doctorat à l'École d'études politiques de l'Université d'Ottawa portant sur la gouvernance globale de la santé et ses enjeux politiques. Il a publié en 2011, L'eau en commun, avec Sylvie Paquerot et Frédéric Julien (Québec, Presses de l'Université du Québec).

$\mathrm{D}$ ans un ouvrage problématisant les fondements philosophiques de la propagation des maladies infectieuses, Jean Lombard et Bernard Vanderwalle rappellent que l'épidémie n'est pas « un moment comme d'autres [...], mais un instant critique ${ }^{1}$ ». Si le conflit étudiant québécois de 2012 a été le vecteur si tenace de la propagation d'un imaginaire social québécois en mutation, avec ses nouveaux héros, ses bons et ses mauvais personnages, ses mises en scène, ses zones d'insécurité et ses traumatismes, c'est que ce conflit représente, au fond, ce type "d'instant critique ", cette zone d'ombres où politique et construit social s'entremêlent et se chevauchent dans une « épidémie de sens ». Ce forum est ainsi l'occasion de revenir sur ce moment polyphonique qualifié par plusieurs commentateurs de "printemps québécois » ou « érable » et qui a pris fin avec l'élection, le 4 septembre dernier, d'un gouvernement minoritaire dirigé par la chef du Parti québécois Pauline Marois, succédant au gouvernement du Parti libéral de Jean Charest qui avait mené les hostilités contre les étudiants.

Le sens des nombres - Le conflit étudiant québécois a tout d'abord été déclenché par une augmentation des frais de scolarité universitaires annuels de 75-82\%, ceux-ci oscillant désormais entre 3793 \$ CA et 3946 \$ CA (entre 3080 et 3200 euros) ${ }^{2}$. C’est une grève générale illimitée initiée le 13 février

1. Lombard J., Vandewalle B., Philosophie de l'épidémie, Paris, L'Harmattan, 2006, p. 16.

2. En fonction des différentes offres du gouvernement, qui ont toutes été rejetées par les associations étudiantes. Avant la hausse, les frais de scolarité universitaires au Québec étaient de 2168 \$ CA, soit environ 1760 euros, ce qui en faisait les moins élevés au Canada, mais parmi les plus élevés des pays de l'OCDE. 
2012 par certaines associations étudiantes et qui, à son paroxysme, a touché plus de $75 \%$ des quelque 400000 étudiants postsecondaires québécois, soit la plus importante grève étudiante de l'histoire du Québec. Ce sont les manifestations du « 22 » de chaque mois qui ont réuni à plusieurs reprises plus de 200000 personnes dans les rues de Montréal, auxquelles s'ajoute une centaine de manifestations nocturnes consécutives. C'est également plus de 3000 arrestations, des milliers de constats d'infraction, des coûts directs et indirects s'élevant à plusieurs centaines de millions de dollars ${ }^{3}$ ainsi que des dizaines de blessés, autant du côté des étudiants que des services de police. Mais le conflit étudiant, c'est avant tout un parti ${ }^{4}$ au pouvoir qui refuse de négocier avec les étudiants et de reconnaitre leur mouvement comme une grève ${ }^{5}$.

Le sens de l'éducation - Le conflit étudiant a également fait naitre un important débat sur la place de l'éducation dans la société québécoise. À la logique d'utilisateur-payeur promue par le gouvernement québécois, à l'instrumentalisation de l'éducation par le marché, le mouvement étudiant québécois a opposé l'éducation comme fondement d'une société qu'il souhaite solidaire et responsable. Contestant la commercialisation de la recherche universitaire et son accaparement par le secteur privé, rejetant une éducation au service de l'économie et non du savoir, de la culture et de l'équité, les étudiants québécois ont défendu le bien commun et la recherche d'un mieux-être collectivement partagé.

Le sens de la mobilisation - Initialement focalisée sur le blocage de la hausse des frais de scolarité, la mobilisation étudiante s'est progressivement transformée en mouvement social abordant une multitude d'enjeux sociétaux. Corruption, environnement, féminisme, santé, droits sociaux, démocratie, etc., sont autant de points d'ancrage qui sont venus cristalliser le débat public autour d'une nouvelle vision d'avenir du Québec. La Coalisation large de l'Association pour une solidarité syndicale étudiante (CLASSE), une des principales associations étudiantes, a même entamé une tournée du Québec intitulée « Nous sommes avenir » dans laquelle elle propose de redéfinir les bases d'un «bien commun " ${ }^{6}$, une expression dont le sens semble échapper au politique au Québec. Un leader étudiant est également devenu candidat pour le

3. Les évaluations divergent, mais certaines estimations des coûts de la grève montrent que ceuxci ont dépassé les gains financiers associés à la hausse des frais de scolarités. Ils ont été évalués par les fédérations étudiantes à plus de 120 millions de dollars canadiens (près de 100 millions d'euros) pour la période de février à avril 2012. Voir http://1625canepassepas.ca/wpcontent/uploads/2012/04/NOTE-ECONOMIQUE.pdf, consulté le 28 juillet 2012.

4. Le parti au pouvoir au Québec durant le conflit étudiant était le Parti libéral du Québec dirigé par le Premier ministre Jean Charest. Il a été remplacé, aux élections du 4 septembre, par un gouvernement minoritaire dirigé par la chef du Parti québécois, Pauline Marois.

5. Le gouvernement de Jean Charest a toujours qualifié le mouvement étudiant de «boycott» et non de grève, car selon lui, les étudiants ne sont pas des «employés » des universités. Le texte de Philipe Langlois, plus loin, revient en détail sur cet élément.

6. "Nous sommes avenir ", manifeste de la CLASSE. Voir www.bloquonslahausse.com/2012/07/nous-sommes-avenir-la-classe-en-tournee-a-traversle-quebec/, consultée le 29 juillet 2012. 
Parti québécois et a été élu le 4 septembre dernier, devenant ainsi le plus jeune député de l'histoire du Québec.

Le sens de la justice - Le conflit étudiant a également placé la sphère juridique au cœur du débat social par l'intermédiaire d'une série d'injonctions des tribunaux dont se sont revendiquées plusieurs centaines d'étudiants pour obtenir le droit d'assister à leur cours, en dépit des mandats de grèves qui avaient été conférés démocratiquement aux associations étudiantes. En porteà-faux vis-à-vis de la démocratie estudiantine, le pouvoir juridique y a joué son indépendance face aux pouvoirs politiques. Le Parlement québécois a également voté le projet de loi spéciale 78 (devenue par la suite la loi 12), limitant fortement la possibilité de mobilisation et de manifestation des citoyens du Québec, ce qui a été vertement critiqué par l'ONU 7, la section canadienne francophone d'Amnesty international (Amnistie internationale) ${ }^{8}$, le Conseil des droits de l'homme ${ }^{9}$, etc. Cette loi s'est toutefois révélée impossible à appliquer tant sa sévérité imposait un contrôle policier, juridique et répressif important ${ }^{10}$, laissant ainsi aux forces de l'ordre le soin de son application arbitraire en fonction de sa perception de l'(il)légalité des manifestations. Dans un élan de paranoïa collective, l'arrestation de certains individus s'est même faite en vertu de dispositions antiterroristes du Code criminel du Québec.

Le sens de la violence - Cette crise étudiante, c'est aussi un gouvernement qui se retourne contre sa propre jeunesse. Une violence qui n'aura de sens que dans la construction médiatique polarisée qui en sera faite d'un côté comme de l'autre. Une violence qui réduira le champ des possibles et rendra vaine l'utilisation de ce conflit comme une interpellation à la prise en charge collective.

Le sens des couleurs - Ce conflit, c'est également une multitude de symboles colorés comme autant de prises de position politiques : les carrés rouges comme emblèmes de la mobilisation étudiante; les carrés bleus pour le refus

7. Voir Matthieu Boivin, "Conflit étudiant : la situation au Québec inquiète l'ONU », La Presse, 31 mai 2012 (www.lapresse.ca/le-soleil/actualites/education/201205/30/01-4530211conflit-etudiant-la-situation-au-quebec-inquiete-lonu.php, consulté le 29 juillet 2012).

8. Amnistie internationale, communiqué de presse : "Conflit étudiant - Amnistie internationale Canada francophone est sérieusement préoccupée par les atteintes au droit de manifester pacifiquement », 23 avril 2012, ainsi que : "Conflit étudiant - Amnistie internationale demande une enquête indépendante sur les interventions policières », 14 mai 2012.

9. Voir «Loi 78 : la situation au Québec est "alarmante", selon l'ONU », Radio Canada, 18 juin 2012 (www.radio-canada.ca/nouvelles/Politique/2012/06/18/003-loi-78-onu.shtml, consulté le 29 juillet 2012).

10. Selon la Loi 12 (anciennement la Loi 78), tout regroupement de plus de 50 personnes dans un lieu public doit fournir à la police un itinéraire de ses déplacements huit heures avant que ceux-ci aient lieu. Ceux qui, individuellement, ne respecteront pas cette disposition pourront se voir infliger des amendes de 1000 à $5000 \$$ canadiens (800-4 000 euros) par jour, un montant qui peut aller jusqu'à $125000 \$$ canadiens (environ 100000 euros) si c'est une association étudiante ou un de ses représentants qui est déclaré coupable. Le montant est doublé en cas de récidive. 
de la hausse et de la grève ; les carrés verts pour la hausse ; les carrés noirs contre la loi 12 ; les carrés blancs pour une trêve.

Le sens du rythme - Ce sont aussi en parallèle des centaines de manifestations de casseroles spontanées à travers tout le Québec qui, dans un clin d'œil à l'histoire des mouvements sociaux latino-américains, a fait résonner le tintamarre d'un ras-le-bol social.

Le sens de l'bumour - C'est même un peu d'humour grinçant. Le Premier ministre Jean Charest par exemple, lors d'une allocution prononcée dans le cadre de la promotion du Plan Nord 11, avait blagué sur les manifestations violentes qui avaient ponctué la journée : «Le Salon du Plan Nord que nous allons ouvrir aujourd'hui, qui est déjà très populaire - les gens courent de partout pour entrer - est une occasion, notamment pour les chercheurs d'emplois. Alors à ceux qui frappaient à notre porte ce matin, on pourra leur offrir un emploi, dans le Nord autant que possible 12 ». Ce à quoi les étudiants ont répondu qu'à la prochaine élection ${ }^{13}$, ce sont eux qui allaient lui trouver un emploi dans le Nord.

En définitive, le conflit étudiant québécois est surtout un rappel du sens politique. Le Québec y a ainsi vu renaître les débats, la discussion, l'opposition, les revendications, la résistance, le désaccord, l'argumentation, la polarisation. Société fondamentalement consensuelle, puisqu'engluée dans une opposition souverainiste/fédéraliste depuis plus de 40 ans, le Québec a redécouvert le sens de l'expression «projet de société » qui, l'espace d'un printemps, est allé au-delà de la revendication de baisses d'impôts. Toutefois, aucun responsable politique ne s'est montré digne de ce mouvement social et politique, se contentant de narratifs comptables sur l'état financier, positif ou négatif selon chacun, du Québec. Cette crise n'aura eu de sens, en définitive, que dans son rappel de l'immaturité des responsables au Québec. C'est la faillite d'une pensée politique dont la date de péremption est échue, c'est l'échec d'une pratique politique qui n'a pas su trouver le courage de ses ambitions. C’est le futur énigmatique d'une nation en gestation. Si le célèbre poète québécois Gilles Vigneault a si bien chanté que le Québec «ce n’est pas un pays, c'est l'hiver ", les étudiants québécois auront eux rappelé que même lorsque c'est le printemps au Québec, c'est toujours l'hiver politique.

11. Le Plan Nord est un projet de développement économique et d'exploitation des ressources naturelles du Nord québécois qui était promu par le gouvernement de Jean Charest et qui visait à investir plus de 80 milliards de dollars canadiens (environ 65 milliards d'euros) sur une période de 25 ans. Voir http://plannord.gouv.qc.ca/index.asp, consulté le 1er août 2012.

12. Voir «Manifestations à Montréal : l'humour de Charest ne passe pas ", Radio Canada, 21 avril 2012, http://www.radio-canada.ca/nouvelles/Politique/2012/04/20/003-etudiantsmanifestation-reax-politique.shtml, consulté le 30 juillet 2012.

13. Les élections générales du Québec, dont le déclenchement reste une prérogative du gouvernement au pouvoir, ont été déclenchées le 1er août 2012 et ont eu lieu le 4 septembre, mettant fin au règne du gouvernement libéral de Jean Charest. Plusieurs voix avaient réclamé la tenue d'élections anticipées pour régler par les urnes le conflit étudiant. 
Ce forum réunit ainsi des textes qui sont autant d'éclairages sur cette situation problématique. Frédéric Julien aborde tout d'abord la crise étudiante sous l'angle d'un choc des idéologies concernant différentes conceptions de l'éducation, des services publics et du processus démocratique. Gabriel Blouin Genest revient quant à lui sur les structures langagières et les narratifs qui ont stimulé l'association entre «manifestations étudiantes » et « violence » durant le conflit, techniques de gouvernance habilement mises en place par le gouvernement libéral de Jean Charest. Philippe Langlois souligne de son côté la façon dont ce gouvernement a tenté de poser le débat relatif à la liberté d'association, qualifiant le mouvement de boycott et non de grève, stigmatisant de la sorte le sens collectif qui a pris forme durant ce conflit et orientant les débats autour d'une conception libérale et individuelle de l'éducation. Enfin, Sylvie Paquerot amène le débat autour de la question de la « juste part », argument qui a été utilisé durant ce conflit pour légitimer la hausse des droits de scolarité au Québec. L'auteure montre ainsi que le recours à cet argument cache un changement d'orientation de société et de compromis social qui est exemplifié par la non-problématisation de la « juste part » des autres acteurs sociaux, et notamment des banques. 